\title{
ODD MULTIPERFECT NUMBERS
}

\author{
SHI-CHAO CHEN ${ }^{\bowtie}$ and HAO LUO
}

(Received 29 May 2012; accepted 16 August 2012; first published online 6 November 2012)

\begin{abstract}
A natural number $n$ is called multiperfect or $k$-perfect for integer $k \geq 2$ if $\sigma(n)=k n$, where $\sigma(n)$ is the sum of the positive divisors of $n$. In this paper, we establish a theorem on odd multiperfect numbers analogous to Euler's theorem on odd perfect numbers. We describe the divisibility of the Euler part of odd multiperfect numbers and characterise the forms of odd perfect numbers $n=\pi^{\alpha} M^{2}$ such that $\pi \equiv \alpha(\bmod 8)$, where $\pi^{\alpha}$ is the Euler factor of $n$. We also present some examples to show the nonexistence of odd perfect numbers of certain forms.
\end{abstract}

2010 Mathematics subject classification: primary 11A05.

Keywords and phrases: odd multiperfect numbers, Euler part, nonexistence.

\section{Introduction}

Let $n$ be a positive integer and $\sigma(n)$ be the sum of the positive divisors of $n$. We say that $n$ is perfect if $\sigma(n)=2 n$ and that $n$ is multiperfect or $k$-perfect if $\sigma(n)=k n$ for some integer $k \geq 2$. For example, 6 is a perfect number and 120 is a 3-perfect number. One can refer to Flammencamp [4] or Hagis and McDaniel [5] for a survey of perfect and multiperfect numbers.

Even perfect numbers were completely classified by Euclid and Euler, but odd perfect numbers remain utterly mysterious: no odd $k$-perfect numbers are known for any $k \geq 2$. But it is known that an odd perfect number must satisfy various conditions. One of the important structural results on odd perfect numbers is due to Euler, who showed that if $n$ is perfect then $n=\pi^{\alpha} M^{2}$, where $\pi$ is prime, $(\pi, M)=1$ and $\pi \equiv \alpha \equiv 1(\bmod 4)$. We call $\pi^{\alpha}$ the Euler factor. Euler's theorem was extended by Broughan and Zhou recently to 4-perfect numbers, and then to $2^{k}$-perfect numbers [1]. In this paper, we will establish a precise result on odd $k$-perfect numbers. It turns out that an odd $k$-perfect number $n$ has the form $n=p_{1}^{e_{1}} p_{2}^{e_{2}} \cdots p_{s}^{e_{s}} M^{2},\left(p_{i}, M\right)=1$, where the odd primes $p_{i}$ and odd positive integers $e_{j}$ satisfy some congruence restrictions.

This research was supported by the Natural Science Foundation of China (Grant No. 11101123) and the Natural Science Foundation of Education Department of Henan Province (Grant No. 2009A110001).

(C) 2012 Australian Mathematical Publishing Association Inc. 0004-9727/2012 \$16.00 
(See Theorem 2.2 for details.) We call $\Pi:=p_{1}^{e_{1}} p_{2}^{e_{2}} \cdots p_{s}^{e_{s}}$ the Euler part of the $k$-perfect number $n$ analogous to the Euler factor of odd perfect numbers.

It is interesting to study the Euler part of $k$-perfect numbers. It turns out that the properties of the Euler part of $k$-perfect numbers can be used to prove the nonexistence of odd $k$-perfect numbers of certain forms. For instance, Starni [11] recently proved that if an odd perfect number $n$ has the form $n=\pi^{\alpha} 3^{2 \beta} Q^{2 \beta}$ with $(3, Q)=1$, then $3^{2 \beta} \mid \sigma\left(\pi^{\alpha}\right)$. As a corollary, he showed that if $\pi \equiv 1(\bmod 12)$ and $\alpha \equiv 1$ or $9(\bmod 12)$, then there do not exist odd perfect numbers $n=\pi^{\alpha} 3^{2 \beta} M^{2}$. This result was extended to odd $2^{k}$-perfect numbers by Broughan and Zhou in [1]. We will show that the prime 3 can be replaced by any Fermat prime under suitable conditions (see Corollary 3.2).

An early result of Starni [10] on odd perfect numbers is that there is no odd perfect number decomposable into primes, all congruent to $1(\bmod 4)$, if $n=\pi^{\alpha} M^{2}$ and $\pi \not \equiv \alpha(\bmod 8)$. In Section 3, we will characterise the forms of odd perfect numbers for which $\pi \equiv \alpha(\bmod 8)$. As a consequence, we extend Starni's results.

\section{Structure of multiperfect numbers}

Recently, Broughan and Zhou [1] generalised Euler's structure theorem on odd perfect numbers to odd 4-perfect numbers, and then to odd $2^{k}$-perfect numbers. To prove the structural result of odd 4-perfect numbers, they employed the following theorem, which is of independent interest.

Theorem (Broughan and Zhou [1, Theorem 2.2]). For all odd primes p, powers $j \geq 1$ and odd exponents $e>0$,

$$
2^{j} \| \sigma\left(p^{e}\right) \quad \text { if and only if } 2^{j+1} \|(p+1)(e+1) .
$$

This theorem was proved by discussing many cases and constructing some polynomials. Here we will give a simple proof. More generally, we have the following theorem.

THeORem 2.1. Let $p$ be a prime and e be a positive integer. Let $v_{2}(m)$ be the highest power of 2 dividing the integer $m$. Then

$$
\begin{aligned}
& v_{2}\left(\sigma\left(p^{e}\right)\right)= \begin{cases}v_{2}(p+1)+v_{2}\left(\frac{e+1}{2}\right) & \text { if } p>2 \text { and } e \equiv 1(\bmod 2), \\
0 & \text { otherwise }\end{cases} \\
& v_{2}\left(\sigma\left(p^{e}\right)-1\right)= \begin{cases}0 & \text { if } p>2 \text { and } e \equiv 1(\bmod 2), \\
v_{2}(p+1)+v_{2}\left(\frac{e}{2}\right) & \text { if } p>2 \text { and } e \equiv 0(\bmod 2), \\
1 & \text { if } p=2 .\end{cases}
\end{aligned}
$$

Proof. It is obvious that $\sigma\left(p^{e}\right)=1+p+\cdots+p^{e} \equiv 1(\bmod 2)$ when $p=2$ or when $p$ is odd and $e$ is even. Now we assume that $p$ and $e$ are both odd. We 
write $r=v_{2}((e+1) / 2)$ and $(e+1) / 2=2^{r} s$ for some odd integer $s$. Then

$$
\begin{aligned}
\sigma\left(p^{e}\right) & =\frac{p^{e+1}-1}{p-1}=\frac{\left(p^{2}\right)^{(e+1) / 2}-1}{p-1} \\
& =\frac{\left(p^{2}\right)^{2^{r} s}-1}{p-1}=(p+1) \frac{\left(p^{2 s}\right)^{2^{r}}-1}{p^{2}-1} \\
& =(p+1)\left(\left(p^{2 s}\right)^{2^{r-1}}+1\right)\left(\left(p^{2 s}\right)^{2^{r-2}}+1\right) \cdots\left(p^{2 s}+1\right) \frac{p^{2 s}-1}{p^{2}-1} .
\end{aligned}
$$

Since $p$ is odd,

$$
\left(p^{2 s}\right)^{2^{r-i}}+1=\left(p^{s 2^{r-i}}\right)^{2}+1 \equiv 2(\bmod 4), \quad 1 \leq i \leq r .
$$

Note that

$$
\frac{p^{2 s}-1}{p^{2}-1}=1+p^{2}+p^{4}+\cdots+\left(p^{2}\right)^{s-1} \equiv s \equiv 1(\bmod 2) .
$$

Therefore

$$
\begin{aligned}
v_{2}\left(\sigma\left(p^{e}\right)\right) & =v_{2}(p+1)+\sum_{i=1}^{r} v_{2}\left(\left(p^{2 s}\right)^{2^{r-i}}+1\right)+v_{2}\left(\frac{p^{2 s}-1}{p^{2}-1}\right) \\
& =v_{2}(p+1)+r \\
& =v_{2}(p+1)+v_{2}\left(\frac{e+1}{2}\right) .
\end{aligned}
$$

Formula (2.2) follows from (2.1) and the fact that

$$
v_{2}\left(\sigma\left(p^{e}\right)-1\right)=v_{2}\left(p \sigma\left(p^{e-1}\right)\right)=v_{2}(p)+v_{2}\left(\sigma\left(p^{e-1}\right)\right) .
$$

This completes the proof of Theorem 2.1.

We remark that Theorem 2.1 can be shown to follow from [9, Theorems 94 and 95].

As an application of Theorem 2.1, we will establish an explicit structure theorem for odd $k$-perfect numbers.

THEOREM 2.2. Let $n$ be odd and $k$-perfect with $v_{2}(k) \geq 1$ and $s$ be any integer satisfying $1 \leq s \leq v_{2}(k)$. Then $n$ has the shape

$$
n=p_{1}^{e_{1}} p_{2}^{e_{2}} \cdots p_{s}^{e_{s}} M^{2}
$$

where $M$ is a positive integer, the $p_{i}$ are primes with $\left(p_{i}, M\right)=1$, and the $e_{j}$ are odd positive integers. If $v_{2}(k)-s$ has a nonnegative partition

$$
v_{2}(k)-s=a_{1}+a_{2}+\cdots+a_{s}+b_{1}+b_{2}+\cdots+b_{s}, \quad a_{i} \geq 0, b_{j} \geq 0,
$$

then the primes $p_{1}, \ldots, p_{s}$ satisfy

$$
p_{i} \equiv 2^{a_{i}+1}-1\left(\bmod 2^{a_{i}+2}\right)
$$


and the exponents $e_{1}, \ldots, e_{s}$ satisfy

$$
e_{j} \equiv 2^{b_{j}+1}-1\left(\bmod 2^{b_{j}+2}\right) .
$$

Before proving the theorem, we give the definition of the Euler part of an odd $k$ perfect number.

Definition 2.3. The Euler part of an odd $k$-perfect number $n$ with the shape (2.3) is denoted by

$$
\Pi:=p_{1}^{e_{1}} p_{2}^{e_{2}} \cdots p_{s}^{e_{s}}
$$

REMARK.

(i) Theorem 2.2 shows that if $n_{1}$ is $k_{1}$-perfect, $n_{2}$ is $k_{2}$-perfect and $v_{2}\left(k_{1}\right)=v_{2}\left(k_{2}\right)$, then $n_{1}$ and $n_{2}$ have the same shapes. Therefore we only consider $2^{k}$-perfect numbers in many situations.

(ii) Note that there are $v_{2}(k)$ shapes of an odd $k$-perfect number $n$ since $s$ can take $v_{2}(k)$ values and each $s$ gives a shape of $n$ as in (2.3).

Proof of Theorem 2.2. Let $n=p_{1}^{e_{1}} p_{2}^{e_{2}} \cdots p_{r}^{e_{r}}$ be the standard factorisation of $n$. Since $n$ is $k$-perfect,

$$
\sigma(n)=\sigma\left(p_{1}^{e_{1}}\right) \sigma\left(p_{2}^{e_{2}}\right) \cdots \sigma\left(p_{r}^{e_{r}}\right)=k n .
$$

Since $v_{2}(k) \geq 1$, it follows from (2.1) of Theorem 2.1 that some $e_{i}$ must be odd. Without loss of generality, we can assume that $e_{1}, \ldots, e_{s}$ are odd and $e_{s+1}, \ldots, e_{r}$ are even. So

$$
n=p_{1}^{e_{1}} p_{2}^{e_{2}} \cdots p_{s}^{e_{s}} \cdot p_{s+1}^{e_{s+1}} p_{s+2}^{e_{s+2}} \cdots p_{r}^{e_{r}}=p_{1}^{e_{1}} p_{2}^{e_{2}} \cdots p_{s}^{e_{s}} M^{2},
$$

where $M^{2}=p_{s+1}^{e_{s+1}} \cdots p_{r}^{e_{r}}$. By Theorem 2.1,

$$
\begin{aligned}
v_{2}(k) & =v_{2}(k n)=v_{2}(\sigma(n)) \\
& =\sum_{i=1}^{s} v_{2}\left(\sigma\left(p_{i}^{e_{i}}\right)\right)+\sum_{j=s+1}^{r} v_{2}\left(\sigma\left(p_{j}^{e_{j}}\right)\right) \\
& =\sum_{i=1}^{s}\left(v_{2}\left(p_{i}+1\right)+v_{2}\left(\frac{e_{i}+1}{2}\right)\right) \\
& =s+\sum_{i=1}^{s}\left(v_{2}\left(\frac{p_{i}+1}{2}\right)+v_{2}\left(\frac{e_{i}+1}{2}\right)\right) .
\end{aligned}
$$

Therefore

$$
v_{2}(k)-s=\sum_{i=1}^{s} v_{2}\left(\frac{p_{i}+1}{2}\right)+\sum_{i=1}^{s} v_{2}\left(\frac{e_{i}+1}{2}\right) .
$$

Given a nonnegative partition of $v_{2}(k)-s$ such that

$$
v_{2}(k)-s=a_{1}+a_{2}+\cdots+a_{s}+b_{1}+b_{2}+\cdots+b_{s}, \quad a_{i} \geq 0, b_{j} \geq 0,
$$

we can take $v_{2}\left(\left(p_{i}+1\right) / 2\right)=a_{i}$ and $v_{2}\left(\left(e_{i}+1\right) / 2\right)=b_{i}, \quad 1 \leq i \leq s$. The desired congruences for $p_{i}$ and $e_{i}$ follow immediately. This completes the proof of Theorem 2.2. 
We give two examples to recover Euler's theorem on odd perfect numbers and Broughan and Zhou's structure theorem on odd 4-perfect numbers [1, Theorem 2.1].

Example 2.4. Let $n$ be an odd perfect number. Then $v_{2}(2)=1, s=1$, and $n$ has the unique form $n=\pi^{\alpha} M^{2}$ with $\pi$ prime, $\alpha$ odd and $(\pi, M)=1$. By (2.4), $a_{1}=b_{1}=0$. So $\pi \equiv \alpha \equiv 1(\bmod 4)$.

ExAmple 2.5. Let $n$ be an odd 4-perfect number. Then $v_{2}(4)=2$ and $s=1$ or 2 .

(1) If $s=1$, then $n=p^{e} M^{2}$. By (2.4), $a_{1}=1, b_{1}=0$ or $a_{1}=0, b_{1}=1$. Therefore $p \equiv 3(\bmod 8), e \equiv 1(\bmod 4)$ or $p \equiv 1(\bmod 4), e \equiv 3(\bmod 8)$.

(2) If $s=2$, then $n=p_{1}^{e_{1}} p_{2}^{e_{2}} M^{2}$. By (2.4), $a_{1}=a_{2}=b_{1}=b_{2}=0$. Therefore $p_{1} \equiv p_{2} \equiv$ $e_{1} \equiv e_{2} \equiv 1(\bmod 4)$.

\section{The Euler part of a $2^{k}$-perfect number}

Based on a result of McDaniel [7], Starni [11] proved that if an odd perfect number $n$ has the form $n=\pi^{\alpha} 3^{2 \beta} Q^{2 \beta}$ with $(3, Q)=1$, then $3^{2 \beta} \mid \sigma\left(\pi^{\alpha}\right)$. This result was generalised to odd $2^{k}$-perfect numbers by Broughan and Zhou [2, Theorem 2.6]. Recall that $\Pi$ is the Euler part of an odd $k$-perfect number. In the following theorem we will prove necessary and sufficient conditions on the divisibility of $\sigma(\Pi)$.

Theorem 3.1. Let $n=\Pi q^{2 \beta} \prod_{i=1}^{s} p_{i}^{2 \beta_{i}}$ be an odd $2^{k}$-perfect number, where $\Pi$ is the Euler part of $n$, and $q$ and the $p_{i}$ are distinct odd primes. Then

$$
q^{2 \beta} \mid \sigma(\Pi) \quad \text { if and only if } \begin{cases}2 \beta_{i}+1 \neq 0\left(\bmod \operatorname{ord}_{q}\left(p_{i}\right)\right) & \text { if } p_{i} \equiv 1(\bmod q), \\ 2 \beta_{i}+1 \neq 0(\bmod q) & \text { otherwise, }\end{cases}
$$

where $\operatorname{ord}_{q}(m)$ is the order of $m$ in the multiplicative group $(\mathbb{Z} / q \mathbb{Z})^{*}$.

Proof. Since $n$ is $2^{k}$-perfect,

$$
2^{k} n=2^{k} \Pi q^{2 \beta} \prod_{i=1}^{s} p_{i}^{2 \beta_{i}}=\sigma(\Pi) \sigma\left(q^{2 \beta}\right) \sigma\left(\prod_{i=1}^{s} p_{i}^{2 \beta_{i}}\right)=\sigma(n) .
$$

Therefore $q^{2 \beta} \mid \sigma(\Pi)$ is equivalent to $\left(q, \sigma\left(p_{i}^{2 \beta_{i}}\right)\right)=1, i=1, \ldots, s$.

Note that if $p_{i} \equiv 1(\bmod q)$, then

$$
\sigma\left(p_{i}^{2 \beta_{i}}\right) \equiv 2 \beta_{i}+1(\bmod q) .
$$

Hence $\left(q, \sigma\left(p_{i}^{2 \beta_{i}}\right)\right)=1$ implies that $2 \beta_{i}+1 \not \equiv 0(\bmod q)$.

If $p_{i} \not \equiv 1(\bmod q)$, then $\left(q, \sigma\left(p_{i}^{2 \beta_{i}}\right)\right)=1$ implies that $p_{i}^{2 \beta_{i}+1} \not \equiv 1(\bmod q)$. It follows that $2 \beta_{i}+1 \not \equiv 0\left(\bmod \operatorname{ord}_{q}\left(p_{i}\right)\right)$. This completes the proof of Theorem 3.1.

Corollary 3.2. Let $n=\Pi q^{2 \beta} \prod_{i=1}^{s} p_{i}^{2 \beta_{i}}$ be an odd $2^{k}$-perfect number as in Theorem 3.1. If $q$ is a Fermat prime, that is, $q=2^{2^{t}}+1$ for some integer $t \geq 0$, and $\prod_{i=1}^{s}\left(2 \beta_{i}+1\right) \not \equiv 0(\bmod q)$, then $q^{2 \beta} \mid \sigma(\Pi)$. 
Proof. Note that $\phi(q)=2^{2^{t}}$, where $\phi$ is the Euler function. Lagrange's theorem implies that $\operatorname{ord}_{q}\left(p_{i}\right) \mid \phi(q)$. Hence $\operatorname{ord}_{q}\left(p_{i}\right)$ is a power of 2. The corollary follows by Theorem 3.1 immediately.

REMARK. If $n=\pi^{\alpha} q^{2 \beta} \prod_{i=1}^{s} p_{i}^{2 \beta}$ is an odd perfect number with $\alpha \equiv \pi \equiv 1(\bmod 4)$, then it is known that $\beta \neq 2[6], \beta \neq 3[5], \beta \neq 5,12,17,24,62[8]$, and $\beta \neq$ $6,8,11,14,18$ [2]. In [8] McDaniel and Hagis conjectured that there do not exist such odd perfect numbers for any positive integer $\beta$. Corollary 3.2 can be used to prove this conjecture in some special cases. For example, if $q=5,(2 \beta+1,5)=1$, $\pi \equiv 1(\bmod 20), \alpha \equiv 13(\bmod 20)$, then by Corollary $3.2,5 \mid \sigma\left(\pi^{\alpha}\right)$, but $\sigma\left(\pi^{\alpha}\right) \equiv 1+\alpha \equiv$ $4(\bmod 5)$. Therefore there do not exist such odd perfect numbers. In particular, $n=41^{13} 5^{2 \beta} \prod_{i=1}^{s} p_{i}^{2 \beta}$ cannot be perfect for any $\beta \not \equiv 2(\bmod 5)$ and odd primes $p_{i}$.

Let $n=\pi^{\alpha} \prod_{i} p_{i}^{2 \beta_{i}}$ be an odd perfect number, $\pi^{\alpha}$ with $\pi \equiv \alpha \equiv 1(\bmod 4)$ being Euler's factor. Based on a result of Ewell [3], Starni [10] proved that $\pi \equiv \alpha(\bmod 8)$ if each prime $p_{i} \equiv 1(\bmod 4)$. In the following Theorem 3.3, we will extend Starni's results independently of Ewell's result.

TheOREM 3.3. Let $n=\pi^{\alpha} M^{2}$ be an odd perfect number, with $\pi$ prime, $(\pi, M)=1$ and $\pi \equiv \alpha \equiv 1(\bmod 4)$. Then

$$
\begin{aligned}
& \sigma\left(M^{2}\right) \equiv 1(\bmod 4) \quad \text { if and only if } \pi \equiv \alpha(\bmod 8), \\
& \sigma\left(M^{2}\right) \equiv 3(\bmod 4) \quad \text { if and only if } \pi \equiv \alpha+4(\bmod 8) .
\end{aligned}
$$

In particular, if $n=\pi^{\alpha} \prod_{j} q_{j}^{2 \gamma_{j}}$ with $q_{j} \equiv 3(\bmod 4)$ then

$$
\pi \equiv \alpha(\bmod 8)
$$

Proof. Using the fact that $\sigma\left(\pi^{\alpha} M^{2}\right)=2 \pi^{\alpha} M^{2}$ and $M$ and $\alpha$ are odd, we find that

$$
\pi^{\alpha} \equiv \pi \equiv \frac{\sigma\left(\pi^{\alpha}\right)}{2} \sigma\left(M^{2}\right)(\bmod 8) .
$$

Note that $\pi \equiv \alpha \equiv 1(\bmod 4)$ implies that $\pi^{4} \equiv \alpha^{4} \equiv 1(\bmod 16)$. It follows that

$$
\begin{aligned}
\sigma\left(\pi^{\alpha}\right) & =1+\pi+\cdots+\pi^{\alpha} \\
& =\left(1+\pi+\pi^{2}+\pi^{3}\right)\left(1+\pi^{4}+\pi^{8}+\cdots+\pi^{\alpha-5}\right)+\pi^{\alpha-1}(1+\pi) \\
& \equiv(1+\pi)\left(1+\pi^{2}\right) \frac{\alpha-1}{4}+1+\pi(\bmod 16) .
\end{aligned}
$$

Hence

$$
\frac{\sigma\left(\pi^{\alpha}\right)}{2} \equiv\left(1+\pi^{2}\right) \frac{1+\pi}{2} \frac{\alpha-1}{4}+\frac{1+\pi}{2} \equiv \frac{1+\pi}{2} \frac{1+\alpha}{2}(\bmod 8) .
$$

Inserting this into (3.3),

$$
\pi \equiv \frac{\pi+1}{2} \frac{\alpha+1}{2} \sigma\left(M^{2}\right)(\bmod 8) .
$$


Since $(1+\pi) / 2$ is odd,

$$
\frac{\pi(\pi+1)}{2} \equiv\left(\frac{\pi+1}{2}\right)^{2} \frac{\alpha+1}{2} \sigma\left(M^{2}\right) \equiv \frac{\alpha+1}{2} \sigma\left(M^{2}\right)(\bmod 8) .
$$

It follows that

$$
\pi(\pi+1) \equiv(\alpha+1) \sigma\left(M^{2}\right)(\bmod 16) .
$$

If $\sigma\left(M^{2}\right) \equiv 1(\bmod 8)$, then

$$
\pi(\pi+1) \equiv \alpha+1(\bmod 16)
$$

Recall that $\pi \equiv \alpha \equiv 1(\bmod 4)$. It is easy to find that the solutions $(\pi, \alpha)(\bmod 16)$ are $(1,1),(5,13),(9,9),(13,5)$. In particular, we get

$$
\pi \equiv \alpha(\bmod 8)
$$

Similar arguments show that the solutions to (3.4) are

$$
(\pi, \alpha)(\bmod 16)= \begin{cases}(1,9) ;(5,5) ;(9,1) ;(13,13) & \text { if } \sigma\left(M^{2}\right) \equiv 5(\bmod 8), \\ (1,5) ;(5,9) ;(9,13) ;(13,1) & \text { if } \sigma\left(M^{2}\right) \equiv 3(\bmod 8), \\ (1,13) ;(5,1) ;(9,5) ;(13,9) & \text { if } \sigma\left(M^{2}\right) \equiv 7(\bmod 8)\end{cases}
$$

This implies (3.1) and (3.2).

If we write $M^{2}=\prod_{i} p_{i}^{2 \beta_{i}} \prod_{j} q_{j}^{2 \gamma_{j}}$ where $p_{i} \equiv 1(\bmod 4), q_{j} \equiv 3(\bmod 4)$, then by (2.2) of Theorem 2.1, $\sigma\left(q_{j}^{2 \gamma_{j}}\right) \equiv 1(\bmod 4)$. Hence

$$
\sigma\left(M^{2}\right)=\prod_{i} \sigma\left(p_{i}^{2 \beta_{i}}\right) \prod_{j} \sigma\left(q_{j}^{2 \gamma_{j}}\right) \equiv \prod_{i}\left(2 \beta_{i}+1\right)(\bmod 4) .
$$

Clearly, if $\beta_{i}=0$ for all $i$, that is $M^{2}=\prod_{j} q_{j}^{2 \gamma_{j}}$, then $\sigma\left(M^{2}\right) \equiv 1(\bmod 4)$, and (3.1) implies that $\pi \equiv \alpha(\bmod 8)$.

Remark. By (3.2), (3.5) and (2.2) of Theorem 2.1, it is easy to see that $\pi \equiv \alpha+$ $4(\bmod 8)$ if and only if the number of prime factors $p^{e}$ of $M^{2}$ with $p^{e} \| M^{2}$, $p \equiv 1(\bmod 4)$ and $e \equiv 2(\bmod 4)$ is odd.

\section{Acknowledgement}

The authors thank the referee for his careful reading of the manuscript and for pointing out reference [9].

\section{References}

[1] K. Broughan and Q. Zhou, 'Odd multiperfect numbers of abundancy 4', J. Number Theory 128 (2008), 1566-1575.

[2] G. L. Cohen and R. J. Williams, 'Extensions of some results concerning odd perfect numbers', Fibonacci Quart. 23 (1985), 70-76. 
[3] J. A. Ewell, 'On the multiplicative structure of odd perfect numbers', J. Number Theory 12 (1980), 339-342.

[4] A. Flammenkamp, 'The multiply perfect numbers page', http://wwwhomes.uni-bielefeld.de/achim/mpn.html.

[5] P. Hagis Jr and W. L. McDaniel, 'A new result concerning the structure of odd perfect numbers', Proc. Amer. Math. Soc. 32 (1972), 13-15.

[6] H.-J. Kanold, 'Untersuchungen über ungerade vollkommene Zahlen', J. reine angew. Math. 183 (1941), 98-109.

[7] W. L. McDaniel, 'The nonexistence of odd perfect numbers of a certain form', Arch. Math. 21 (1970), 52-53.

[8] W. L. McDaniel and P. Hagis Jr, 'Some results concerning the nonexistence of odd perfect numbers of the form $p^{\alpha} M^{2 \beta}$, Fibonacci Quart. 13 (1975), 25-28.

[9] T. Nagell, Introduction to Number Theory (AMS Chelsea Publishing, Providence, RI, 1981).

[10] P. Starni, 'On the Euler's factor of an odd perfect number', J. Number Theory 37 (1991), 366-369.

[11] P. Starni, 'On some properties of the Euler's factor of certain odd perfect numbers', J. Number Theory 116 (2006), 483-486.

SHI-CHAO CHEN, Institute of Contemporary Mathematics, Department of Mathematics and Information Sciences, Henan University, Kaifeng 475001, PR China

e-mail: schen@henu.edu.cn

HAO LUO, Institute of Contemporary Mathematics, Department of Mathematics and Information Sciences, Henan University, Kaifeng 475001, PR China e-mail: luohao200681@126.com 